\title{
Data Driven Fuel Efficient Driving Behavior Feedback for Fleet Vehicles
}

David Gertman, Dumidu Wijayasekara, Milos Manic

July 2015

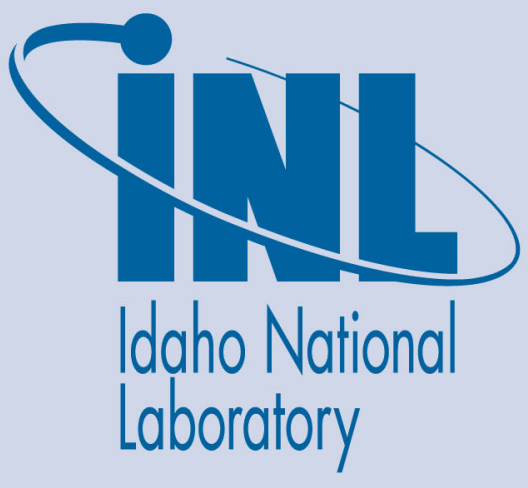

The INL is a U.S. Department of Energy National Laboratory operated by Battelle Energy Alliance 


\title{
Data Driven Fuel Efficient Driving Behavior Feedback for Fleet Vehicles
}

\author{
David Gertman, Dumidu Wijayasekara, Milos Manic
}

July 2015

Idaho National Laboratory Idaho Falls, Idaho 83415

http://www.inl.gov

Prepared for the U.S. Department of Energy

Office of Energy Efficiency and Renewable Energy

Under DOE Idaho Operations Office

Contract DE-AC07-05ID14517 


\section{Data Driven Fuel Efficient Driving Behavior Feedback for Fleet Vehicles}

\author{
Dumidu Wijayasekara, Milos Manic \\ Virginia Commonwealth University \\ Richmond, Virginia, USA \\ dumidu.wijayasekara@gmail.com, misko@ieee.org
}

\author{
David Gertman \\ Idaho National Laboratory (INL) \\ Idaho Falls, Idaho, USA \\ david.gertman@inl.gov
}

\begin{abstract}
Dependency of the transport sector on fossil fuels is encouraging a significant amount of research in to improving fuel efficiency in vehicles. Three primary techniques are identified for vehicle fuel efficiency improvement: 1) vehicle technology improvements such as drivetrain improvements, 2) traffic infrastructure improvements such as traffic flow management and route selection, and 3) driver behavior changes such as acceleration and deceleration profiles. Out of the 3 techniques, driver behavior changing has the least implementation cost and is able to provide immediate results. Thus, this paper presents a fuel efficient driving behavior identification and feedback architecture that is specific to fleet vehicles. The presented method utilizes historical data from fleet drivers on specific routes and generates fuel optimal velocity profiles that do not affect travel time. The identified velocity profile is the prompted to the driver via a low-cost plug-and-play style un-obstructive display. The display uses an intuitive and easily understandable visualization to prompt drivers on fuel efficient velocity. The presented architecture was tested on the Idaho National Laboratory (INL) bus fleet in real-world driving conditions and was shown to be able to increase the fuel economy by $9 \%$ and $20 \%$ in two different driving scenarios.
\end{abstract}

Keywords-Eco-driving; visualization; fuel efficiency; driver feedback; passive driver assistance

\section{INTRODUCTION}

With increasing fossil fuel prices and the climate change concerns related to carbon emissions and other pollutants, reducing fossil fuel usage is gaining increasing attention. Research has shown that the transportation sector is the largest consumer of fossil fuels in many countries. For example nearly $71 \%$ of the petroleum consumed in the United States (U.S.) is consumed by the transportation sector [1], [2]. Furthermore, it has also been shown that the transportation sector is the largest contributor to carbon emissions throughout the world. In the $\mathrm{EU}$, transportation is attributed to more than a fourth of carbon emissions [3]. Similarly, in the U.S. the transportation sector is the largest carbon emitter contributing to over a third of all carbon emissions, of which over $40 \%$ are from passenger cars [4]. Furthermore, in the U.S. the carbon emissions by the transportation sector has increased in recent years [4].

Therefore, increasing vehicle fuel efficiency has become a major research area with significant impacts on fossil fuel usage and the global carbon footprint [5], [6], [7]. Three major techniques are identified in the literature for improving vehicle fuel economy, which are: 1) vehicle technology improvements, 2) traffic infrastructure improvements, and 3) driver behavior changes [8].

Vehicle technology improvements entail improving the physical design of the vehicle such as engine, gearbox, and aerodynamics [7], [9], [10]. Alternative fuel designs and improvements fall under this category as well. While, these improvements have the highest potential for long term impacts, they have long implementation times, high implementation cost, and will not affect the vehicles currently on the road [5], [11].

Traffic infrastructure improvements are concerned with managing traffic flow such that vehicle idle times and travel times are reduced. These methods further include alternate route selection with an emphasis on fuel economy [8]. While, traffic infrastructure improvements yield immediate results and affects all road vehicles, they are costly to implement and logistically difficult.

Driver behavior changes related to fuel economy entail an overall less aggressive driving style, with smooth acceleration and deceleration profiles [3], [12], [13], [14]. Such driving techniques are known as "eco-driving" and can be loosely defined as a driving decision making process that positively influences the vehicle fuel efficiency [3]. Thus, driver behavior changes do not require any mechanical changes or infrastructure changes, which makes it easy to implement with comparatively very low cost [5], [12], [15]. Furthermore, they can be implemented in already existing vehicles, leading to fast implementation and turn-around times [5], [12]. Previous studies report fuel efficiency improvements ranging in 5-15\% through driver behavior changes alone [3], [13]. Thus, this paper is concerned with changing driver behavior to achieve improved fuel economy.

Two primary methods of influencing driver behavior for improved fuel economy has been investigated in the past: 1) driver education, 2) driver feedback. Driver education entails training drivers on fuel efficient driving styles [12], [15]. While this method has been shown to be effective [1], [15], it has been shown that over time, some drivers tend to depart from fuel efficient behavior [15], [16]. Furthermore, training a fleet of drivers is time consuming and costly. Driver feedback entails real-time or non-real-time feedback about the fuel economy and how to change behavior for increased fuel 
economy. Real-time continuous feedback devices are known as passive driving assistance tools and have been shown to the extremely effective [17], [18].

The primary concern of real-time feedback devices is safety [5]. While audio [18], [19], visual and haptic [20] feedback for fuel efficient driving has been investigated in the past, it can be argued that visual feedback is the optimal. Visual feedback that are non-distracting and easily ignored ensures safe operation [20]. For example audible cues may be distracting in hazardous situations where quick decisions need to be made. Furthermore, early research has suggested that drivers may have up to $50 \%$ spare attention capacity in regular driving conditions [21]. Recent research have further suggested that glancing behavior at an in-vehicle information system suggests that it may not lead to visual distraction, and might not increase mental workload [22].

This paper presents a fuel optimal driver behavior identification and feedback architecture for fleet vehicles driving in preset highway routes. Fleet vehicles present a unique situation where different drivers drive similar vehicles over a preset route where the driving times are governed by strict guidelines [6]. Furthermore, highway driving conditions are more consistent and have less aggressive acceleration and deceleration points. The presented architecture uses a modified version of the data driven driver behavior identification methodology presented in [6], and utilizes the low cost, easy to understand prompting framework along with the visualization previously presented in [5]. The presented architecture was tested on a real-world driving scenario with 3 drivers on a 12 kilometer route in Idaho. The experimental results show 9\% and $20 \%$ increase in the average fuel economy with the presented framework implemented.

This paper is organized as follows; section II details the data driven driver behavior identification and extraction methodology, and the driver behavior feedback framework is detailed section III. Section IV provides the implementation details while section V presents experimental results. Finally, section VI presents final conclusions and future research directions.

\section{FUEL EFFICIENT DRIVER BEHAVIOR IDENTIFICATION}

This section describes the driver behavior identification methodology for fleet vehicles that is used in this paper. The driver behavior identification methodology is a slightly modified version of the method that was presented in [6].

As mentioned, fleet vehicles present a unique situation where similar vehicles are being driven on the same route multiple times by different drivers. Thus, the basic idea behind the driver behavior identification methodology is to utilize driving data from multiple drivers to derive an overall fuel optimal behavior for a specific route. The final extracted behavior will be a combination of driving behavior of multiple drivers, driving similar vehciles, multiple times on the same route. Furthermore, the presented method is for highway driving situations, and it is assumed that the vehicle will be in the same transmission gear position throughout the run. This was found to be a safe assumption by analyzing the data for the specific route chosen for the experiment.

In order to combine multiple data from multiple drivers, first data is gathered from multiple runs on the same route in similar vehicles. The data is collected from the vehicle onboard diagnostics system, can be represented as:

$$
d(t)=\{v(t), p(t), f(t), \ldots\}
$$

where, $d(t)$ is the data point collected at time $t$. $v(t), p(t)$, and $f(t)$ are the velocity, the position, and the fuel rate of the vehicle and at time $t$ respectively. Other features such as transmission gear position, engine rpm, and gas pedal position are also collected, but will not be used for generating the driver behavior profile.

The state of the vehicle at time $t, x(t)$ is extracted from the collected data and can be expressed as:

$$
x(t)=[v(t), p(t)]
$$

where $v(t)$, and $p(t)$ are the velocity, and the position of the vehicle and at time $t$ respectively. Thus, for an entire run the state of the dataset can be expressed as: $X=\{x(t), t=0 \ldots T\}$, where $T$ is the total data collection time.

However, in order to synchronize multiple runs according a specific route, the temporal data must be converted in to spatial domain. Thus, using $p(t)$, the state of the vehicle at position $p, x(p)$ can be expressed as:

$$
x(p)=[v(p), p]
$$

where, $v(p)$ is the velocity of the vehicle at position $p$.

Thus the converted spatial dataset can be expressed as $X_{P}=\{x(p), p=0 \ldots L\}$, where $L$ is the length of the route.

Once the data is converted in to the spatial domain, segments can be identified. These segments are defined in terms of a starting position $p_{S}$ and an end positon $p_{E}$. Thus a segment can be expressed as $S\left(p_{S}, p_{E}\right)$. To combine data from multiple datasets, identical segments will be used, meaning the state of the vehicle in each dataset at $p_{S}$ and $p_{E}$ must be identical. Thus given two datasets in the spatial domain $X_{P 1}$ and $X_{P 2}$, a segment that can be merged $S_{M}\left(p_{S}, p_{E}\right)$ can be identified by:

$$
\begin{aligned}
S_{M}\left(p_{S}, p_{E}\right) \Rightarrow x_{1}\left(p_{S}\right)=x_{2}\left(p_{S}\right) \wedge & \\
& x_{1}\left(p_{E}\right)=x_{2}\left(p_{E}\right)
\end{aligned}
$$




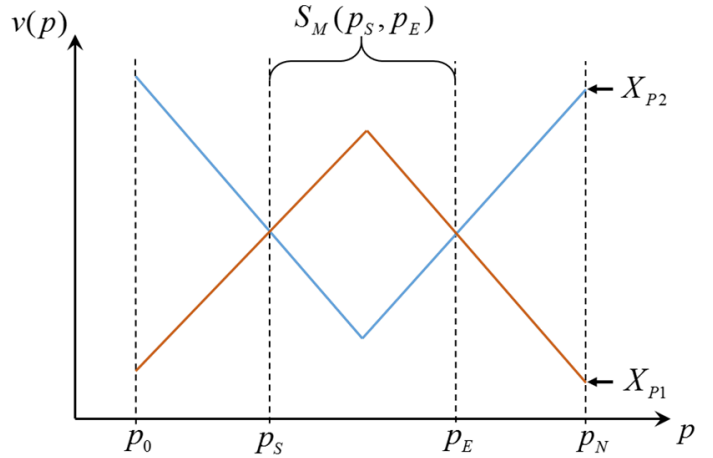

(a)

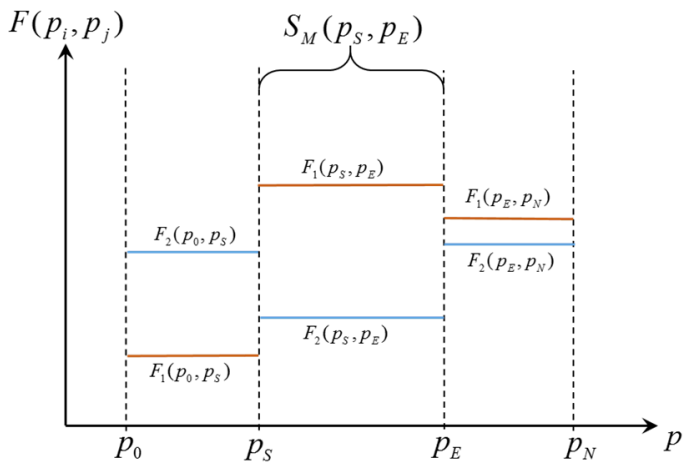

(b)

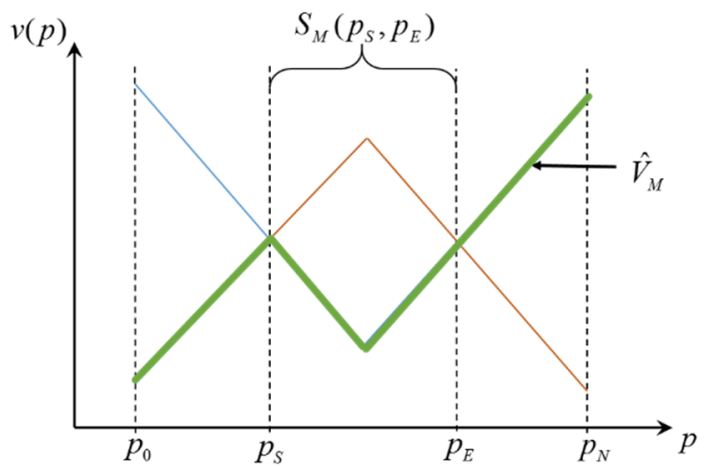

(c)

Fig. 1 The process of extracting the fuel efficient driving behavior from two datasets. (a) identifying segments that can be merged, (b) calculation of the cumulative fuel usage for each segment, (c) identifying the most fuel efficient velocity profile

where, $x_{1}(p) \in X_{P 1}$ and $x_{2}(p) \in X_{P 2}$. The set of points that satisfy these conditions are called switch points (see Fig. 1(a)).

Thus, the definition of $S_{M}\left(p_{S}, p_{E}\right)$ ensures that segments from different datasets, can be interchanged without affecting the physical feasibility of the final solution [6]. The two datasets $X_{P 1}$ and $X_{P 2}$ can be from two different drivers driving similar vehicles on the same route or the same driver driving the same vehicle on the same route at two different times.

Using the recorded fuel rate $f(t)$, the fuel rate for a given position $f(p)$, can be extracted. The amount of fuel consumed by the vehicle for a given segment $S\left(p_{S}, p_{E}\right)$ can then be calculated as:

$$
F\left(p_{S}, p_{E}\right)=\int_{p_{S}}^{p_{E}} f(p) d p
$$

where, $F\left(p_{S}, p_{E}\right)$ is the amount of fuel consumed between positions $p_{S}$ and $p_{E}$.

The amount of fuel used at each segment can then be used to merge 2 given runs to generate a single velocity profile that is more fuel efficient than the initial runs. The merged velocity profile $V_{M}\left(p_{S}, p_{E}\right)$ for two datasets $X_{P 1}$ and $X_{P 2}$ at a segment that can be merged $S_{M}\left(p_{S}, p_{E}\right)$ can be expressed as:

$$
V_{M}\left(p_{S}, p_{E}\right)=\left\{\begin{array}{cc}
V_{1}\left(p_{S}, p_{E}\right) & \text { if } F_{1}\left(p_{S}, p_{E}\right) \\
& <F_{2}\left(p_{S}, p_{E}\right) \\
V_{2}\left(p_{S}, p_{E}\right) & \text { otherwise }
\end{array}\right.
$$

where, $V_{i}\left(p_{S}, p_{E}\right)=\left\{v_{i}(p), p=p_{S} \ldots p_{E}\right\}$ for a given run $i$.

Note that for the starting segment $S_{0}\left(0, p_{i}\right)$ and for ending segment $S_{L}\left(p_{j}, L\right)$ there may not be any segment that meets the requirements in (4), in these special cases equation (6) is used even though the merging criteria is not met. Thus, the final merged velocity profile $\hat{V}_{M}$ can be expressed as:

$$
\hat{V}_{M}=V_{0}\left(0, p_{i}\right) \cup V_{M}\left(p_{i}, p_{i+1}\right) \cup V_{L}\left(p_{C}, L\right)
$$

where, $V_{0}$ and $V_{L}$ are the start and end segment velocity profiles respectively, and $i=1 \ldots(C-1)$ where, $C$ is the number of switch points for the two datasets given. The process of merging two datasets with two switch points is detailed in Fig. 1.

However, given multiple datasets, finding the most optimal fuel efficient velocity profile is not trivial since switch points are different in different datasets. Furthermore, while minimizing the fuel efficiency, the time to destination should be kept within an acceptable level, as well as maintaining the velocity of the vehicle within certain legal and safe bounds. Thus, the problem of combining multiple datasets to generate a single velocity profile with the optimal fuel efficiency is a bounded multi-criteria optimization problem. While there are many different methods of finding the optimal defined by these parameters, in this paper, a simple evolutionary algorithm was used. 


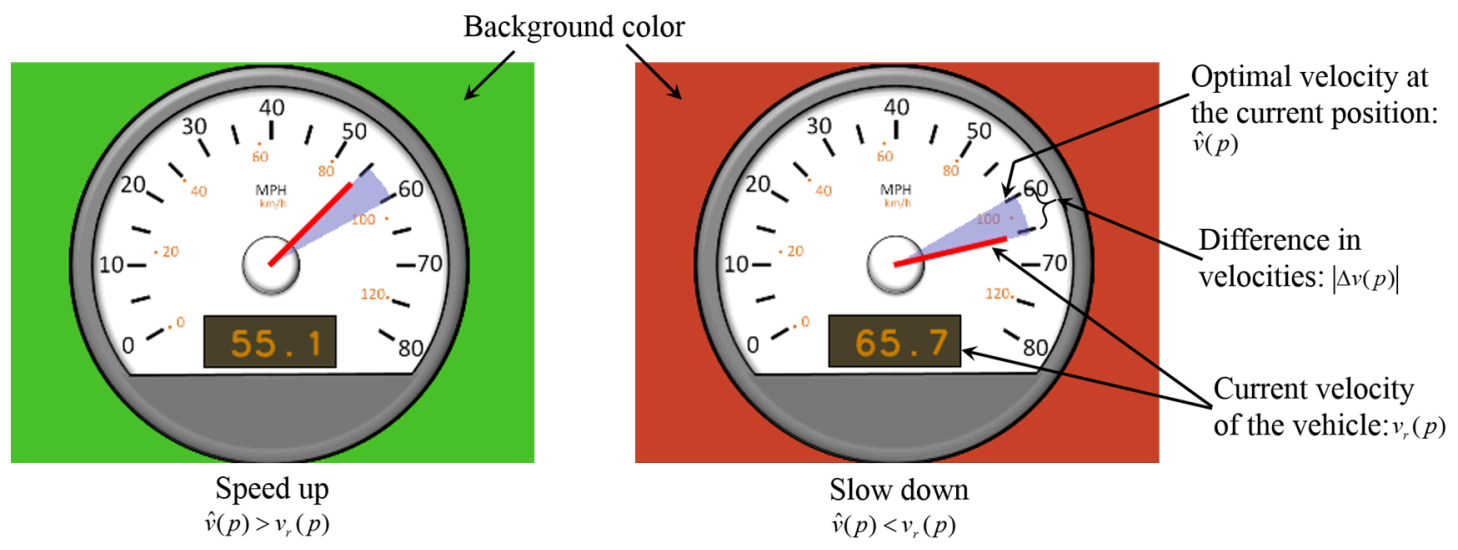

Fig. 2 Visualization of the fuel efficient velocity on the speedometer

The final fuel efficient driver behavior extracted from the process mentioned above is a set of velocities of the vehicle for a given position of the route. This set of velocities is only optimal to the specific route and type of vehicle. The velocity profile can be improved as more data becomes available for that route.

\section{DRIVER BEHAVIOR PROMPTING}

The extracted fuel efficient driving behavior is recorded in terms of a set of velocities for a given positions in the route. This information should be prompted to the driver real-time in an unobtrusive manner.

For prompting the fuel efficient velocity, the framework presented in [5] was used. In this framework, real-time data is gathered from the vehicle along with location information and utilized to provide accurate and timely feedback to the driver

The vehicle related data such as velocity, engine rpm, and transmission gear is collected via the on-board diagnostics systems available in modern vehicles [5]. These systems collect accurate, high frequency data from sensors throughout the vehicle for diagnostics purposes and can be readily collected via industry standard interfaces.

The road position data can be collected via accurate GPS devices. Such devices are becoming increasingly available as the technology progresses. However, to increase the accuracy of the positioning, a combination of prior knowledge about the route and the real-time vehicle speed is used to augment the latitude and longitude information from the GPS device [5]. This type of dead-reckoning can be used to maintain accurate position information even during loss of GPS data. Thus, combining data from the vehicle and the GPS device a data point of the vehicle at time $t, d(t)$ can be created (see equation (1)).

Once the data is collected, as before, the temporal data is converted to the spatial domain and the real-time state of the vehicle $x_{r}(p)$ at a given position $p$ is generated:

$$
x_{r}(p)=\left[v_{r}(p), p\right]
$$

where, $v_{r}(p)$ is the real-time velocity of the vehicle at position $p$.

Using the merged optimal fuel profile generated in the previous section, $\hat{V}_{M}$, the optimal velocity for the current position $p, \hat{v}(p)$ can be found. However, since merged data is discrete, optimal velocity for the current position might not be available. In such cases, linear interpolation of the optimal velocities between the two closest available positons $\hat{V}_{M}$ will be used as the optimal velocity.

Once $v_{r}(p)$ and $\hat{v}(p) \quad$ is calculated, $\Delta v(p)=v_{r}(p)-\hat{v}(p)$ is also calculated. The driver is then presented with this information using a visual display.

The visual display setup and the visualization that is used in this paper is the visualization that was found to be least obstructive, most intuitive, and most understandable in [5]. The visualization used is shown in Fig. 2. The speedometer dial in the visualization is a near exact match to the actual speedometer in the vehicle. Furthermore, the current velocity of the vehicle is clearly displayed. Thus, drivers who are accustomed to looking at the speedometer frequently can utilize the provide visualization to get velocity information of the vehicle. The current actual velocity of the vehicle is augmented with a colored segment that shows the difference in the current velocity of the vehicle and the fuel efficient velocity for the current position $\Delta \hat{v}(p)$. Furthermore, the background color changes from green to red representing whether the current velocity is lower than optimal or higher than optimal, respectively. The intensity of the color represents the magnitude of the difference $|\Delta v(p)|$.

\section{IMPLEMENTATION}

This section details the hardware implementation of the presented fuel optimal driver behavior identification and feedback architecture. The presented architecture was 


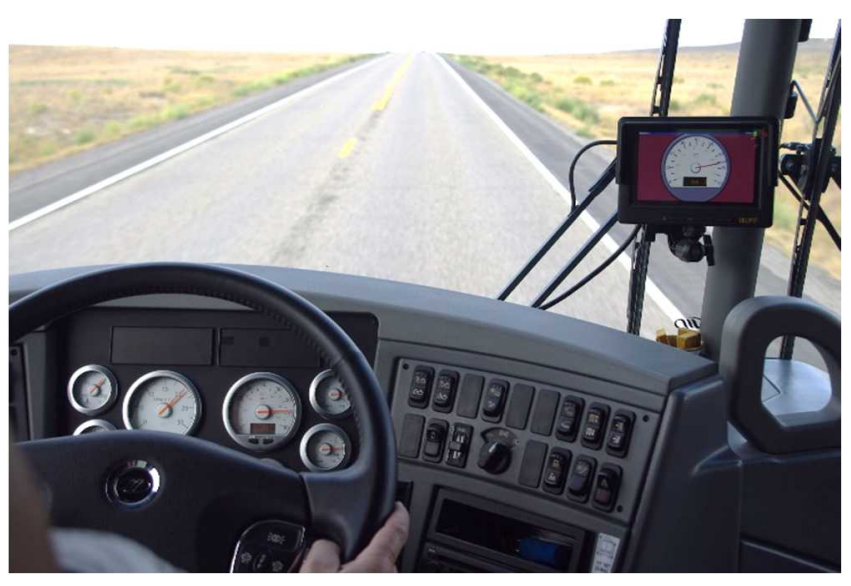

Fig. 3 Presented visualization placed in the bus cabin, in the driver's periphery

implemented on the Idaho National Laboratory (INL) bus fleet using low-cost Commercial Off-The-Shelf (COTS) hardware.

The INL bus fleet consists of over 90 buses of several models that travel in several preset routes throughout the southeastern region of Idaho [23]. For the implementation of the presented framework, the MCI D-series model D4505 buses were selected [24].

The hardware implementation of the data collection and visualization was similar to the previously presented setup in [5]. Since the optimal velocity calculation can be performed offline, the amount of processing that need to be performed real-time is minimal. Thus a small, low power computing device is sufficient for the real-time calculations. A low cost, self-powered USB enabled tablet device running Windows operating system was therefore utilized as the on-board data processing computer.

Data collection for fuel efficient driver behavior identification and real-time behavior prompting was performed through the industry standard system called CANbus, utilized by the MCI D4505 buses. For diagnostic purposes, a 6 pin Deustch connection is present in the cabin of the bus. Using a commercially available interface device called the Nexiq ${ }^{\mathrm{TM}}$ USB link [25], a USB equipped computer can be interfaced with the CANbus via the Deustch connector. Thus, the raw data being passed through the CANbus can be read by the computer. However, the raw data is encoded in using the J1939 protocol [26], which is decoded in the computer to access the actual data stream [5].

For obtaining the position data, a low cost, USB enabled, COTS device from US Global Sat Inc. was used [27]. As, with the raw data from the bus, a separate data parser was used to decode the data stream from the GPS device and obtain the actual latitude and longitude data. Because of the low resolution of the GPS device and the high probability of errors, a combination of dead-reckoning using the velocity of the bus from the Nexiq device and prior knowledge about the route was used in conjunction with the GPS data for accurate positioning.

In order to present the driver with the visualization described in Section III, a 7 inch High-Definition display (

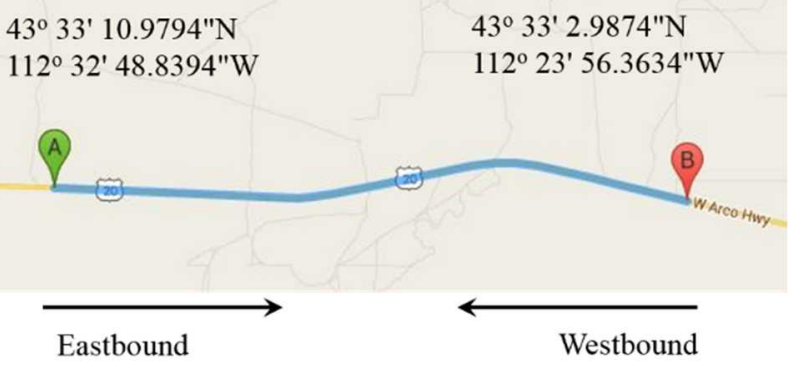

(a)

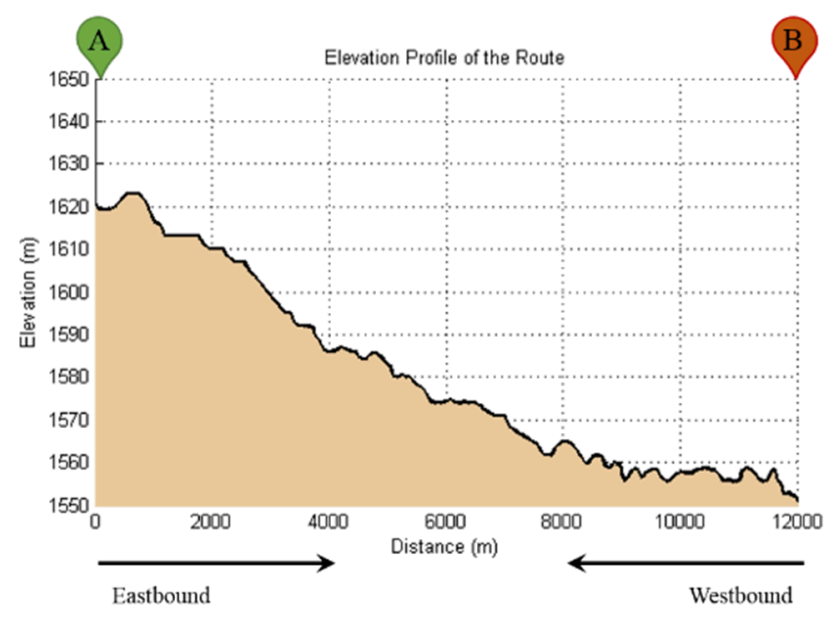

(b)

Fig. 4 Selected $12 \mathrm{~km}$ route (a) top view (b) elevation profile

$1280 \times 720$ resolution) display was utilized. The display was self-powered and mountable, so that it can be placed anywhere in the drivers' periphery that is least obstructive while being easy to glance at. The high resolution and high brightness $\left(450 \mathrm{~cd} / \mathrm{m}^{2}\right)$ of the device maximized the legibility of the visualization. The small form factor and portable nature of the device ensured that the device could be easily positioned within the bus cabin (See Fig. 3).

\section{EXPERIMENTAL RESULTS}

In order to test the presented architecture, a $12 \mathrm{~km}$ portion of the US20 West highway in eastern Idaho was selected. This portion of the highway was selected because: 1 ) ease of access to buses from town, 2) consistent traffic conditions, 3) access to turning points for buses, 4) varying elevation profile and 5) one of the most used routes of the buses. Fig. 4(a) shows the selected portion of the route and Fig. 4(b) shows the elevation profile. Starting point (point A in Fig. 4) was set at lat. $43^{\circ} 33^{\prime}$ $10.9794 " \mathrm{~N}$, long. $112^{\circ} 32^{\prime} 48.8394^{\prime \prime} \mathrm{W}$ and finishing point (point B in Fig. 4) was set at lat. $43^{\circ} 33^{\prime} 2.9874 " \mathrm{~N}$, long. $112^{\circ}$ $23^{\prime} 56.3634 " \mathrm{~W}$. Two sets of data were collected for the selected portion of the route: 1) Eastbound: travelling from point A to point $\mathrm{B}, 2)$ Westbound: travelling from point $\mathrm{B}$ to point $\mathrm{A}$. 


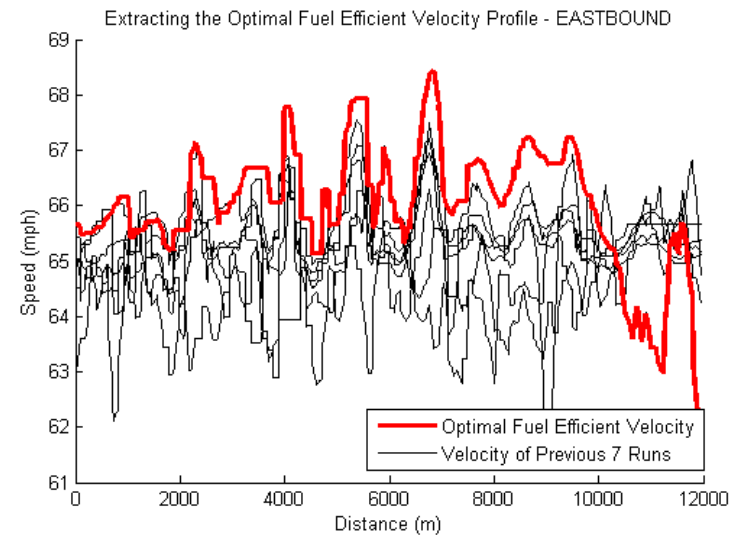

(a)

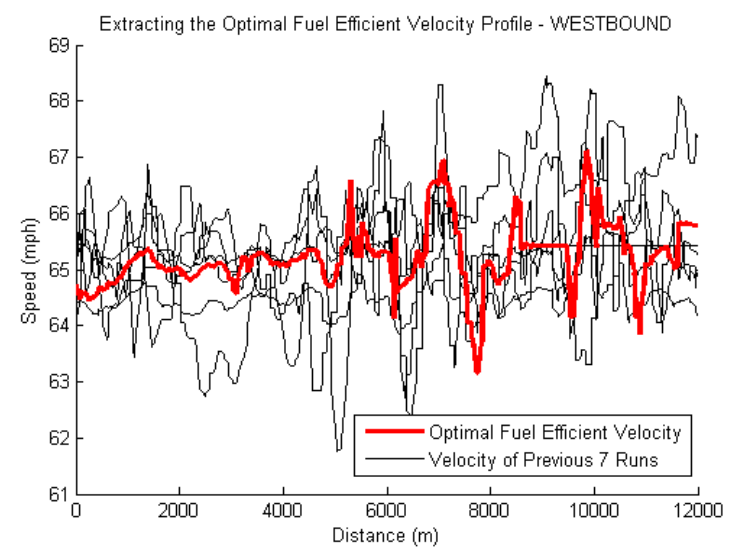

(b)

Fig. 5 Extracted optimal fuel efficient velocity profile (a) Eastbound (b) Westbound

The data was collected using a single bus and three different drivers. First, data was collected to extract the optimal fuel efficient driving behavior. Thus, each driver was first asked to drive the route in a manner which they judged to be the most economical, while keeping the bus within $5 \mathrm{mph}$ of the speed limit (65mph). Each driver was given 3 runs in each direction. In order to keep the data uniform and relevant, weather conditions were monitored during the data collection and due to unfavorable weather, 2 runs were removed from the Eastbound direction. To keep the datasets uniform, the Westbound runs corresponding to the removed runs were also removed. Thus, 7 runs in each direction was used to extract the fuel efficient driver behavior. Fig. 5(a) and 5(b) depict the extracted velocity profiles for Eastbound and Westbound directions respectively.

Once the fuel efficient driver behavior was extracted, the presented visualization framework was setup in the bus and the same drivers were asked to drive the routes while the prompter is running. The drivers were specifically asked to keep attention on the road as usual and only pay attention to the prompter if they feel the necessity, and the conditions allow it. Due to time and resource constraints only 5 runs in each direction were performed with the prompter running $(2$ drivers performing 2 runs and the other driver performing only 1 run, in each direction).

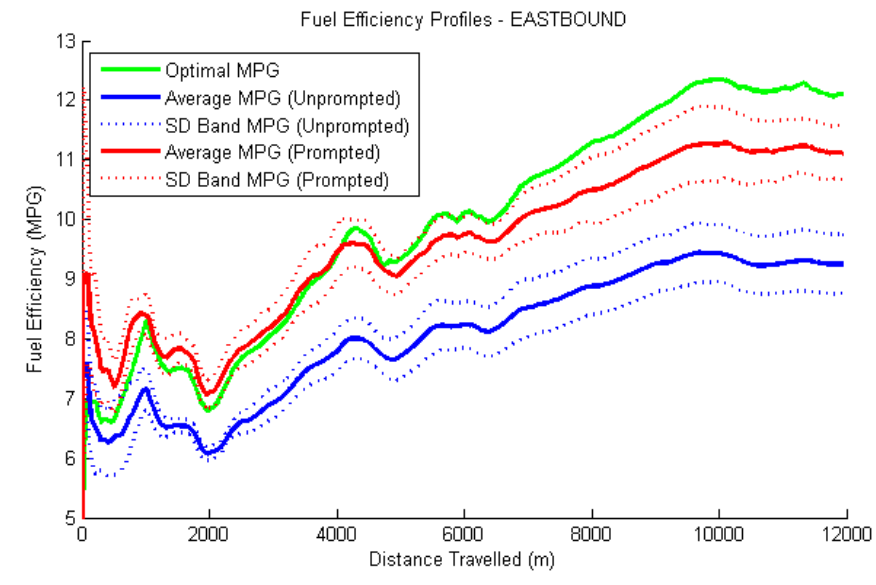

(a)

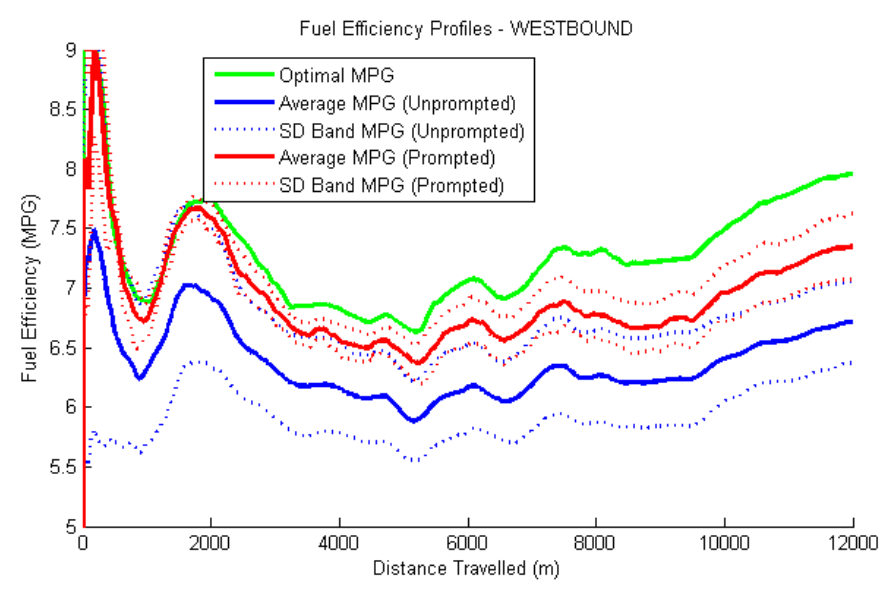

(b)

Fig. 6 Average fuel efficiency curves (a) Eastbound (b) Westbound

TABLE I

AVERAGED FUEL EFFICIENCY FOR EACH RUN

\begin{tabular}{c|c|c|c|c}
\cline { 2 - 5 } & \multicolumn{2}{c|}{ Unprompted } & \multicolumn{2}{c}{ Prompted } \\
\cline { 2 - 5 } & $\begin{array}{c}\text { Average } \\
(\mathrm{mpg})\end{array}$ & $\begin{array}{c}\text { SD } \\
(\mathrm{mpg})\end{array}$ & $\begin{array}{c}\text { Average } \\
(\mathrm{mpg})\end{array}$ & $\begin{array}{c}\text { SD } \\
(\mathrm{mpg})\end{array}$ \\
\hline \hline Eastbound & 9.25 & 0.49 & 11.12 & 0.46 \\
\hline Westbound & 6.72 & 0.35 & 7.34 & 0.28 \\
\hline \hline
\end{tabular}

Fig. 6(a) and 6(b) show the average and standard deviation bands of the fuel efficiency of each run along with the extracted optimal fuel efficiency for Eastbound and Westbound runs respectively. The final fuel efficiencies are given in Table I. From the initial experimental results it can be observed that with the presented architecture operating, the average fuel economy has been increased and has a lower variability, in both cases. However, the lower standard deviation in the prompted case can be attributed to the smaller sample size.

For the Westbound run travelling slightly uphill, the average fuel economy was increased by $9 \%$ while for the slight downhill Eastbound direction the increase was 20\%. In both 
cases the difference in fuel economy with and without the presented architecture was statistically significant with a $95 \%$ confidence interval.

For a pessimistic estimation of fuel economy, the best fuel economy achieved without the prompting and the worst fuel economy with the prompting were compared. For the Eastbound direction, these numbers were nearly identical with the unprompted showing $0.4 \%$ better fuel economy. The same numbers for the Westbound direction showed an increase of $5 \%$ fuel economy with prompting. Thus even with the most pessimistic comparison, the fuel economy with the presented architecture was at least as good as without it.

\section{CONCLUSIONS}

The need to reduce fossil fuel usage has been exemplified in recent years with increasing oil prices and the need to reduce the carbon footprint. Transportation sector has been shown to be the highest consumer of fossil fuel throughout the world and also has the most potential of improving. One of the least expensive and easy to deploy methods of achieving better fuel economy in vehicles is passive driver assistance devices.

Thus, this paper presented an architecture for fleet vehicles for identifying and extracting fuel efficient behavior of drivers and presenting drivers with a continuous feedback on the most fuel efficient velocity through an un-obstructive, easy to understand visualization. The presented architecture was implemented using low-cost COTS devices on a MCI D-series bus, and tested in real-world driving conditions.

The experimental results showed $9 \%$ and $20 \%$ improvements on average fuel consumption using the presented architecture on two different tests. The differences achieved were shown to be statistically significant in both cases when compared to the presented architecture not being used.

Future works entails further experimentation with longer runs and larger number of drivers to obtain more generalized results. By implementing the system for a longer period of time it will be possible to identify driver acceptance of the system and longer term effects. Furthermore, driver distraction and glace frequencies with and without the system should be measured to identify safety risks involved.

\section{ACKNOWLEDGMENT}

This manuscript is the result of work conducted under sponsorship by Battelle Energy Alliance, LLC under Contract No. DE-AC07-05ID14517 with the U. S. Department of Energy. The authors would like to thank support given by Mr. Scott Wold, Manager for the Idaho National Laboratory (INL) Mission Support Services, and Jeffrey Brown and the professional bus drivers who helped make this effort a success.

\section{REFERENCES}

[1] Inventory of the U.S. Greenhous Gas Emssions and Sinks: 1990-2010, U.S. Environmental Protection Agency, 2012.

[2] S. C. Davis, S. W. Diegel, R. G. Boundy, "30th Edition Transportation Energy Data Book", Oak Ridge National Laboratory report, 2011.

[3] Md. S. Alam, A. McNabola, "A critical review and assessment of EcoDriving policy \& technology: Benefits \& limitations," in Transport Policy, vol. 35, pp. 42-49, Sept. 2014.
[4] Inventory of the U.S. Greenhous Gas Emssions and Sinks: 1990-2013, U.S. Environmental Protection Agency, Apr. 2015.

[5] D. Wijayasekara, M. Manic, D. Gertman, "Driving Behavior Prompting Framework for Improving Fuel Efficiency," in Proc. of IEEE Int. Conf. on Human System Interaction, (HSI), June, 2014.

[6] O. Linda, M. Manic, "Improving Vehicle Fleet Fuel Economy via Learning Fuel Efficient Driving Behavior," in Proc. of IEEE Int. Conf. on Human System Interaction, (HSI), June, 2012.

[7] S. D. Cairano, D. Bernardini, A. Bemporad, I. V. Kolmanovsky, "Stochastic MPC With Learning for Driver-Predictive Vehicle Control and its Application to HEV Energy Management," in IEEE Trans. on Control Systems Technology, July 2013.

[8] R. J. T. G. Thijssen, T. Hofman, J. Ham. "Ecodriving acceptance: An experimental study on anticipation behavior of truck drivers," in Transportation research part F: traffic psychology and behaviour, vol. 22, pp. 249-260, 2014.

[9] X. Ma, "Towards intelligent fleet management: Local optimal speeds for fuel and emissions," in Proc. IEEE Int. Conf. on Intelligent Transportation Systems (ITSC), pp. 2201-2206, Oct. 2013.

[10] A. D. Alvarez, F. S. Garcia, J. Naranjo, J. Anaya, F. Jimenez, "Modeling the driving behavior of electric vehicles using smartphones and neural networks," in IEEE Intelligent Transportation Systems Magazine, vol. 6, no. 3, pp. 44-53, 2014.

[11] M. van der Voort, M. Dougherty, "Reducing fuel consumption by using a new fuel-efficiency support tool," in IEE Africon, vol. 1, pp. 27-32, 1999

[12] J. N. Barkenbus, "Eco-driving: An overlooked climate change initiative," in Energy Policy, vol. 38, no. 2, pp. 762-769, 2010.

[13] S. Birrell, J. Taylor, A. McGordon, J. Son, P. Jennings, "Analysis of three independent real-world driving studies: A data driven and expert analysis approach to determining parameters affecting fuel economy," in Transportation research part D: transport and environment, vol. 33, pp. 74-86, 2014.

[14] D. J. Chang, E. K. Morlok, "Vehicle speed profiles to minimize work and fuel consumption," in Journal of Transportation Engineering, vol. 131, pp. 173-182, Mar. 2005.

[15] B. Beusen, S. Broekx, T. Denys, C. Beckx, B .Degraeuwe, M. Gijsbers, K. Scheepers, L. Govaerts, R. Torfs, L. I. Panis, "Using on-board logging devices to study the longer-term impact of an eco-driving course," in Transportation Research Part D: Transport and Environment, vol. 14, no. 7, pp. 514-520, 2009.

[16] C. C. Rolim, P. C. Baptista, G. O. Duarte, T. L. Farias, "Impacts of onboard devices and training on Light Duty Vehicle Driving Behavior," in Procedia-Social and Behavioral Sciences, vol. 111, pp. 711-720, 2014.

[17] M. E. Rakauskas, J. S. Graving, M. P. Manser, J. W. Jenness, "Determining the Accuracy and Acceptance of Using Driver Interface Display Components and Fuel Economy Information Types," in Proc. of the Human Factors and Ergonomics Society Annual Meeting, vol. 54, no. 19, pp. 1536-1540, 2010.

[18] C. Rolim, P. Baptista, G. Duarte, T. Farias, Y. Shiftan, "Quantification of the Impacts of Eco-driving Training and Real-time Feedback on Urban Buses Driver's Behaviour," in Transportation Research Procedia, vol. 3, pp. 70-79, 2014.

[19] Q. Li, F. Qiao, L. Yu, "Will Vehicle and Roadside Communications Reduce Emitted Air Pollution?," in International Journal of Science and Technology, vol. 5, no. 1, 2015.

[20] S. L. Jamson, D. L. Hibberd, A. H. Jamson, "Drivers' ability to learn eco-driving skills; effects on fuel efficient and safe driving behaviour," in Transportation Research Part C: Emerging Technologies, 2015.

[21] P. Hughes, B. Cole, "What attracts attention when driving?," in Ergonomics, vol. 29, pp. 377-391, 1986.

[22] S. A. Birrell, M. Fowkes, "Glance behaviours when using an in-vehicle smart driving aid: A real-world, on-road driving study," in Transportation research part F: traffic psychology and behaviour, vol. 22, pp. 113-125, 2014.

[23] Idaho National Lab Bus / Fleet / Fuel Transformation [Online] Available: http://energy.gov/sites/prod/files/INL\%20Coach\%20Bus\%20Brief.pdf 
[24] Motor Coach Iindustries, 2014 MCI - D-Series [Online] Available: http://www.mcicoach.com/luxury-coaches/passengerDseries.htm

[25] Nexiq Technologies, Nexiq USB-link [Online] Available: http://www.nexiq.com/USBLink2
[26] SAE J1939 Standard for Serial control and communication [Online] Available: http://standards.sae.org/j1939_201206/

[27] US GlobalSat Inc. [Online] Available: http://www.usglobalsat.com/ 